\title{
Application of Method for Calculating Sky View Factor Using Google Street View: Relation Between Sky View Factor and Physical Elements in Urban Space
}

\author{
Shoko NISHIO *, Fumiko ITO \\ Tokyo Metropolitan University, Department of Urban Science and Policy, snishio@tmu.ac.jp, itofumi@tmu.ac.jp \\ * Corresponding author
}

\begin{abstract}
We applied a computation method of calculating the sky view factor (SVF) using Google Street View to Shibuya area, Tokyo, for the purpose of examining the relation between the SVF/SVF change and physical elements. The distribution of the SVF calculated by the above method was visualized, and the statistical process showed the tendency of a high SVF in quasi-residential districts and roadsides of high-graded trunk roads. The difference in the SVF change was small at 10-m intervals. The SVF change tended to be more apparent near an intersection and at different elevations.
\end{abstract}

Keywords: sky view factor, Google Street View, sky view factor change, zoning

\section{Introduction}

\subsection{Background}

With the remarkable development of computers and the Internet in recent years, the use of big data has entered various fields, including medicine, education, transportation, energy, housing, and urban development. Google Street View (GSV) can be regarded as an open big dataset, and its images can be retrieved using an application programming interface (API). GSV is a service that was released on May 25, 2007 in the United States by Google, and it offers $360^{\circ}$ panoramic views. Although only major cities were available initially, panoramic street views from numerous places are now obtainable. The streets can be viewed $360^{\circ}$ horizontally and $290^{\circ}$ vertically from each point via the web. In addition, the option of zoom is available, and the perspective can be moved approximately $10 \mathrm{~m}$ forward or backward from the current point. The original image that provides a view is a panoramic image with the range mentioned above. Each image is associated with the latitude and longitude at the street location in consecutive $10-\mathrm{m}$ intervals, and the images exist as massive data on the web. We proposed a computation method of calculating the sky view factor (SVF) using GSV (Nishio et al., 2014). The SVF is defined as the ratio of the area of the sky in a fisheye image.

The use SVF was introduced as a concept for easing the restrictions on oblique lines when the Building Standards Acts in Japan was revised in 2003. ${ }^{1}$ The amount of the

\footnotetext{
${ }^{1}$ The Building Standards Acts in Japan is a Japanese law that provides the minimum standard regarding the site, facilities,
}

sky in a hemispherand is contained in an equisolid angle projection. The SVF is related to the feelings of a person (Takei et al, 1977; Nishio et al, 2015), and it is one of the important structural elements when considering a comfortable city to live in. Generally, a software such as JWCAD is used to calculate the SVF. Furthermore, in previous studies, a digital camera with a fisheye lens equipped to produce a fisheye image was used (Takei et al, 1977; Nishio et al, 2015). This method is effective for accurately calculating the SVF at a single point. Contrastingly, the Ministry of Environment (2003) calculates the SVF by modeling the building arrangement in a 500-m mesh. The above-mentioned methods are effective for understanding the distribution of the SVF over a large scale. In recent years, studies regarding the calculation of the SVF over a large scale, and in detail, have been frequently seen, such as those by Liang et al (2017) and Zeng et al (2018). These studies use deep learning methods, and the sky area can be extracted correctly than before. The method of equisolid angle projection was used for converting a panoramic image to a fisheye image.

\subsection{Objective}

In this study, we use a method (Nishio et al.,2014) that acquires a GSV image and performs image processing with a computer to calculate the SVF. The SVF is associated with the latitude and longitude information for the considered street. We applied the computation method above to the Shibuya area, Tokyo, for examining the relation between the SVF/SVF change and physical elements.

structure, and use of buildings to protect people's lives, health, and property. 


\subsection{Novelty}

The novelty of this study is two-fold. The first novelty is the clarification of the relation between the SVF distribution and physical elements in an urban space in regarding the city plan rule. Among the prior research that analyzed an urban space using GSV, some studies focused on streetscape and landscapes. One of these studies focused on using a panoramic photo without converting its projection method. Asada et al. (2016) developed a method for landscape visualization using panoramic images from GSV and analyzed the regional landscape based on the spatial distribution of the road landscape. One prior study by Li et al. (2017) quantified and mapped the openness of street canyons by applying a geometric transformation and image classification to the GSV panoramas. Middel et al. (2018) retrieved GSV photos and converted them into hemispherical views by an equiangular projection to analyze an urban climate. In contrast, the current research focused on the analysis of urban areas using GSV images, as explained above. We calculated the SVF from GSV images and analyze the relation between the SVF and city form, particularly delivered based on urban planning rules and the road width. The second novelty is to analyze the change in the SVF. The GSV images are linked by the latitude and longitude and have a positional relation. The position relations are composed of network data, which makes it possible to calculate the change in the SVF. Nishio et al. (2015) reported that the subjects felt open and active ${ }^{2}$ in those areas of a city that had a high SVF. Moreover, the feelings of a person are influenced by the environment while walking. Nishio et al. (2015) argued that the subjects felt open and active while walking when the SVF at the current location was higher than that at a location $10 \mathrm{~m}$ before the current location. The GSV images were photographed at approximately $10-\mathrm{m}$ intervals. Therefore, it is possible to visualize changes in the SVF in detail using our method.

\subsection{Definition of SVF}

\subsubsection{Definition of SVF in this paper}

In this paper, SVF is defined as the ratio of the amount of visible sky to that of the entire sky at an arbitrary measurement location, as shown in an equisolid angle projection. There are two differences from its general definition. The first is related to the term "visible sky", which is the portion of the sky that only includes the building to be constructed according to the Building Standards Acts in Japan. Here, we define it as the ratio of the visible sky to the entire sky while including all the buildings, plants, telephone poles, and signs. The second difference is related to the measurement location. Because the SVF is calculated from GSV images, the measurement location is the center of the road at a height of $2.45 \mathrm{~m}$ or $2.05 \mathrm{~m}$. As described above, the SVF addressed in this research is different from that defined in the Building Standards Acts in Japan. We use the definition provided in previous studies.

\footnotetext{
${ }^{2}$ Feelings such as motivation to walk and pleasantness
}

\subsubsection{SVF calculation using a fisheye lens}

We compared the SVF calculated using GSV with that calculated using a fisheye lens to verify the consistency. The images were photographed using a converter (Nikon Fisheye Converter FC-E8) attached to a compact digital camera (Nikon COOLPIX 4500) and tripod (SLIK PRO 330DXII) in the equidistant projection at each measurement location. They were converted to an orthograph using SPCONV ${ }^{3}$ to calculate the SVF.

\section{Method for Calculating SVF}

\subsection{Overview of the Calculation Method}

This method consists of two programs: (1) a program that acquires the latitude, longitude, and PanoID information from GSV, and (2) a program that acquires and saves the corresponding panoramic image with its PanoID and calculates the SVF by image processing (Figure 1). PanoID is a unique number that is assigned to each $360^{\circ}$ panoramic image in GSV. We implemented program (1) in a web browser, which could be used to acquire and generate a list of the PanoID, latitude, and longitude of the location where the panoramic image was photographed. Program (2) then acquired the corresponding panoramic image via a Mercator projection with the PanoID, performed edge detection via image processing, and created binary images of the area of the sky and other areas. The image was subsequently converted to an orthograph, and the ratio of the visible sky to the entire sky was calculated. Automating these steps makes it possible to calculate the SVF at $10-\mathrm{m}$ intervals over a large scale. Details of the above methods are described in the next section.

\subsection{Creation of Latitude, Longitude, and PanoID List (Program 1)}

The following specifications are implemented in a web browser using Google Maps API V3 with HTML and JavaScript $^{4}$. The latitude, longitude, and PanoID are described at the bottom of the browser screen when an arbitrary point is clicked. Additionally, a specification that repeats the description of the GSV points automatically and continuously is implemented. The point whose information is already acquired is stored in memory to avoid acquiring it from the same point. The latitude, longitude, and PanoID are described in each sentence at the bottom of the browser screen and copied to a text file for further analysis.

\subsection{Image Acquisition and Sky Detection ((1) and (2) in Program 2)}

A panoramic image $(512 \times 512$ pixels $)$ was acquired, and the area necessary for the SVF calculation $(416 \times 110$ pixels) was cut automatically using Python to generate a PanoID. The panoramic image at the location (RGB color) was converted to a grayscale image to aid image processing. Edge detection of the sky was subsequently

\footnotetext{
${ }^{3}$ http://news-sv.aij.or.jp/kankyo/s12/Resource/ap/SPCONV/SP CONV.html

${ }^{4}$ http://www.comp.tmu.ac.jp/fiweb/itoken/nishi/panoindex.html
} 


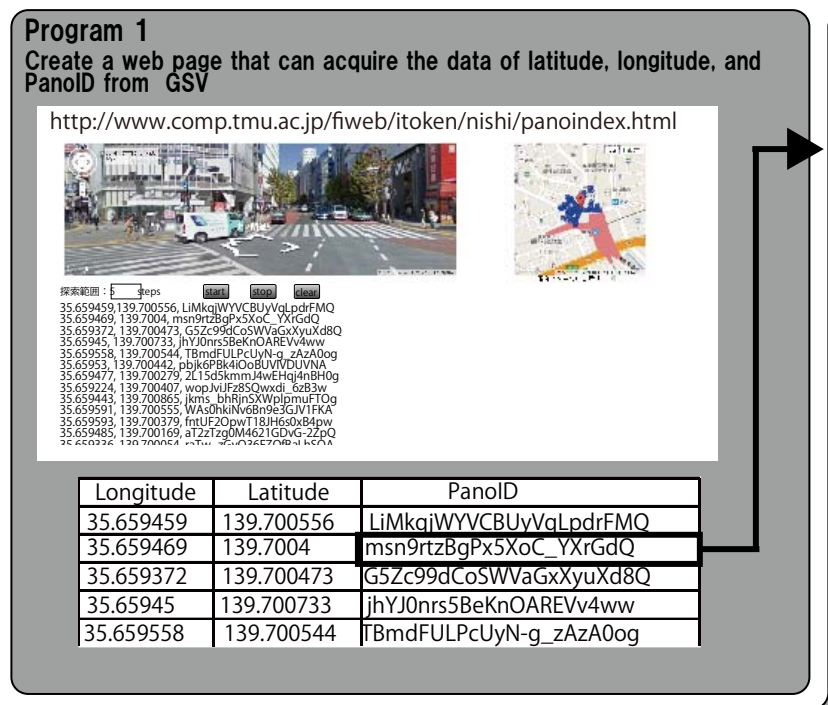

(4) Calculate the SVF
Calculate the percentage of the number of the pixels
in the sky area (white) to the one in the whole area (circle)
$67.2 \%$ for this example
(5)Add to the list
\begin{tabular}{|l|l|l|c|}
\hline Longitude & Latitude & \multicolumn{1}{|c|}{ PanolD } & SVF \\
\hline 35.659459 & 139.700556 & LiMkgjWYVCBUyVqLpdrFMQ & 37.0718073 \\
\hline 35.659469 & 139.7004 & msn9rtzBgPx5XoC_YXrGdQ & $\mathbf{6 7 . 2 0 9 3 4 2 1}$ \\
\hline 35.659372 & 139.700473 & G5Zc99dCoSWVaGxXyuXd8Q & \\
\hline 35.65945 & 139.700733 & jhYJOnrs5BeKnOAREVv4ww & \\
Go back to & $(1)$ to calculate the next PanolD \\
(G5Zc99dCosWVaGXXyuXd8Q in this example)
\end{tabular}

Figure 1. Overview of the SVF calculation method

performed using a brightness threshold with the Canny algorithm in OpenCV. The edge was dilated, and a process called closing, which erodes the dilated edge, was conducted because it prevented the edge from disconnecting from areas of the sky and other areas. Thus, the edge between areas of the sky and other areas was detected, and a binary image was created to detect the sky.

\subsection{Conversion of Projection Method and Calculation of SVF ((3), (4), and (5) in Program 2)}

The panoramic image was photographed using a Mercator projection. The projection of the fisheye image was an orthographic projection in which the image displayed on the hemispherand was projected longitudinally. We discuss the height of the cylinder. We defined the length of the panoramic image as $h$, the width as $w$, and the circumference of the cylinder as $w$. The height of the cylinder was defined as $h$ because the ratio of the height and width appeared to be $1: 1$ in the panoramic image. Because Google has not revealed the actual ratio, it is necessary to define it carefully by comparing the converted fisheye image with that photographed with a fisheye lens; thus, there is some scope for further consideration.

The equation for converting a panoramic image to a fisheye image is explained as follows. First, we defined arbitrary coordinates on the panoramic image as $(X, Y)$, the corresponding point on the fisheye image as $r \sin \theta$ in polar coordinates, and the radius of the hemispherand as

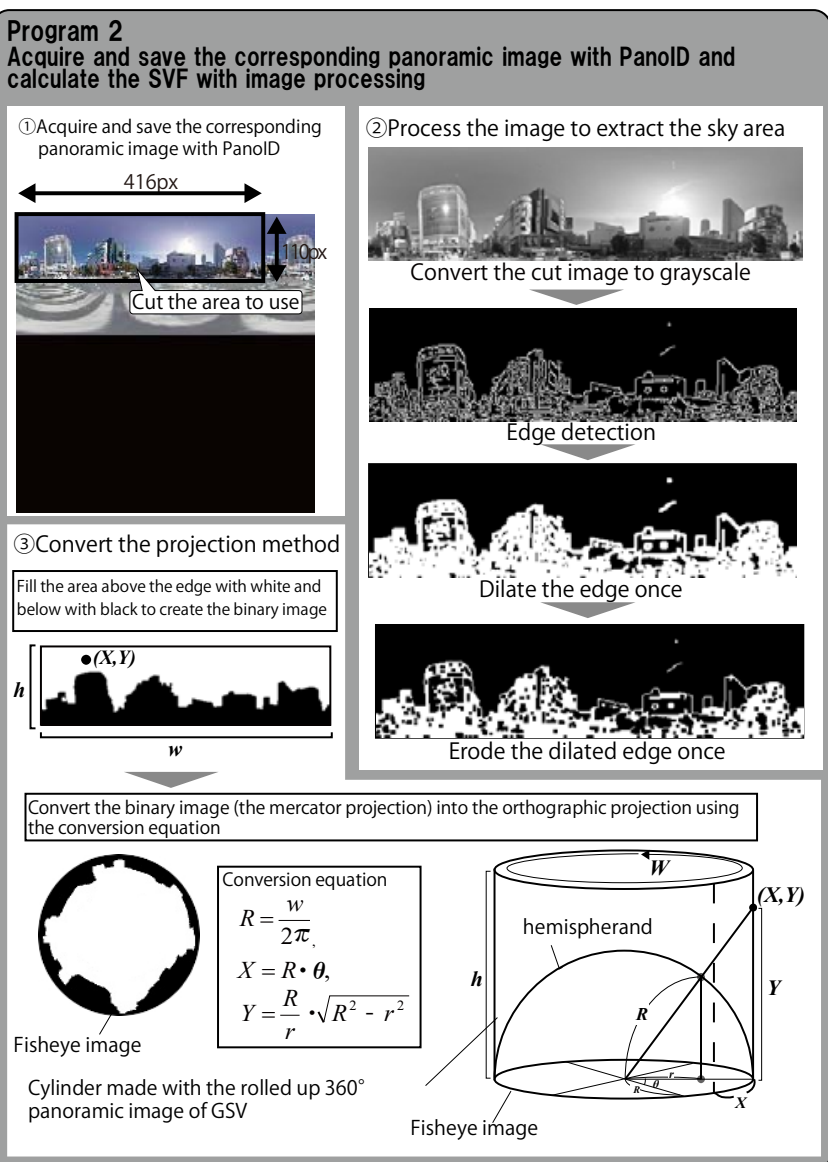

$R$. The following relations are confirmed between the combination of $X$ and $Y$ and that of $r$ and $\sin \theta$ :

$$
R=\frac{w}{2 \pi}, \quad X=R \cdot \theta, \quad Y=\frac{R}{r} \cdot \sqrt{R^{2}-r^{2}}
$$

The SVF was calculated by determining the entire area (circle) in the fisheye image converted using the above equation. The above steps output a list of the latitude, longitude, PanoID, and SVF for the acquired data.

\subsection{Consistency Analysis}

In this section, the accuracy of the SVF calculated with the method is discussed.

First, we discuss the conversion of the projection method (3) in Program 2). Points A and B are provided as examples. We postulate the fisheye image photographed with the fisheye lens is true, and calculate the SVF. We then compare the SVF calculated using the method with that obtained using the fisheye image to confirm to what extent the true SVF is reproduced. At point A, the SVF calculated from the fisheye image photographed with the fisheye lens is $66.9 \%$ and with image processing is $67.2 \%$, yielding an error of $0.3 \%$ (Figure 2). At point B, the SVF calculated from the fisheye image photographed with the fisheye lens is $50.8 \%$ and with image processing is $52.9 \%$ yielding an error of $2.1 \%$ (Figure 2 ).

Second, we discuss the method for detecting the sky (2) in Program 2). We considered 20 randomly selected points. First, the image from each point acquired from 
GSV was cut $416 \times 110$ pixels (left row in Figure 3). Second, the cut image was visually checked and painted in terms of the sky and other areas using Photoshop to create a binary image (central row in Figure 3). The area of the sky was painted in white, whereas the other areas were painted black, and the projection method was then converted to calculate the SVF. The SVF calculated with the above steps was defined as true. The difference between the true SVF and that calculated using the above method was subsequently determined. These steps were repeated at the 20 randomly selected points. First, at 11 points, the error in the SVF is $5 \%$ or less, which can be considered as successful and accurate sky detection (e.g., Point 4 in Figure 3). Then, six points have an error in the SVF ranging from $5 \%$ to $15 \%$ (e.g., Point 12 in Figure 3 ). These points have an overhead line, and it is considered that the error occurs because even if the sky is visible in the area between the overhead line and a building, it is not processed as the sky. Finally, at three points, the error in the SVF is $15 \%$ or more (e.g., Point 20 in Figure 3). Because obstructions are present, such as the overhead line and clouds, almost no area is recognized as the sky at the top of the image at these points in the binary image generated using the method. The mean value of the true SVF calculated using the painted binary image was $55.39 \%$, whereas the SVF calculated using the method was $47.88 \%$, and the error between the SVFs was $7.85 \%$.

We plan to improve the method by using other specifications, such as machine learning, as suggested by Liang et al. (2017), Zeng et al. (2018), and Middel et al.

(2018). We proceed to the following analysis because the true values were calculated at approximately half of the

\begin{tabular}{|l|c|c|}
\hline $\begin{array}{c}\text { Point } \\
\text { Number }\end{array}$ & $\begin{array}{c}\text { Fisheye images photographed } \\
\text { with the fisheye lens }\end{array}$ & $\begin{array}{c}\text { Fisheye images created with } \\
\text { image processing }\end{array}$ \\
\hline $\mathrm{A}$ & $66.9 \%$ \\
\hline $\mathrm{B}$ & & \\
\hline
\end{tabular}

Figure 2. Analysis of the projection method

\begin{tabular}{|c|c|c|c|}
\hline \multirow{2}{*}{ Point4 } & Images cut from GSV & Visually created binary images & Binary images created using the method \\
\hline Point12 & & &
\end{tabular}

Figure 3. Method for extracting sky (Data from Points 4, 12, and 20 are shown as examples)

${ }^{5}$ Shibuya City is a unique ward in the Tokyo prefecture. While it is considered a tourist site and business district, it also has vast tracts of green land, such as the Yoyogi park.

${ }^{6}$ Ratio of the building area to the site area

${ }^{7}$ Ratio of the total floor-area of buildings to the site area 


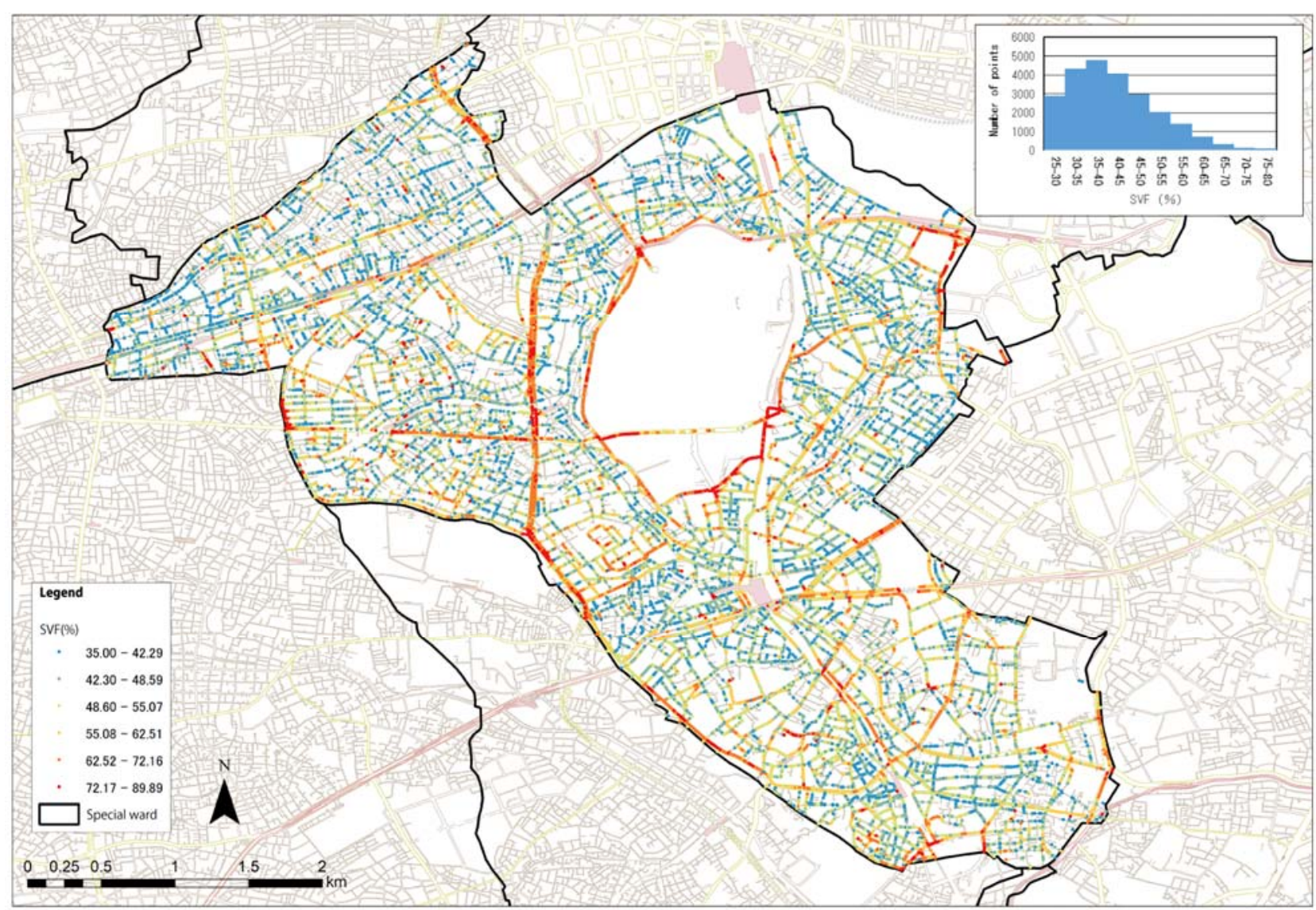

Figure 4. Visualization and histogram of the SVFs in Shibuya city

the industrial districts nor exclusive industrial districts exist in Shibuya city, they are omitted in the following analysis. Figure 5 shows the land-use zoning in Shibuya city on a map.

The SVF values in the entire Shibuya City were aggregated by land-use zoning. A box plot is shown in Figure 6. The following can be seen: the distribution of the residential use in all the districts has median and mean values ranging from approximately $45 \%$ to $65 \%$. Among these, the mean SVF value is the highest in the quasi-residential districts. Regarding the districts for commercial use, there is no significant difference in between the neighborhood commercial districts and commercial districts. The mean value and median are similar for Category 2 low-rise exclusive residential districts and commercial districts. Category 2 low-rise exclusive residential districts are the areas where low-rise buildings are lined on narrow roads. Contrastingly, commercial districts have wide roads and are lined with medium-rise buildings, which is one of the factors for the similar mean and median values.

\subsubsection{Discussion}

First, the highest mean SVF value was found in quasiresidential districts; we believe this was because the districts are located along relatively wide roads. Within Shibuya city, the quasi-residential districts are located along Yamanote-dori Street (shown in Figure 5, hereinafter called quasi-residential district(1)) and to its west (shown in Figure 5, hereinafter called quasi-residential

\begin{tabular}{|c|c|c|c|c|}
\hline & Land-use Zoning & $\begin{array}{l}\text { Building } \\
\text { coverage } \\
\text { ratio }\end{array}$ & \begin{tabular}{|l|}
$\begin{array}{l}\text { Floor } \\
\text { area ratio }\end{array}$ \\
\end{tabular} & $\begin{array}{l}\text { Maximum } \\
\text { number of } \\
\text { stories }\end{array}$ \\
\hline \multirow{10}{*}{ 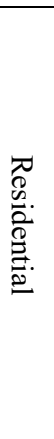 } & $\begin{array}{l}\text { Category } 1 \text { low-rise exclusive } \\
\text { residential districts }\end{array}$ & 60 & 150 & 2.50 \\
\hline & $\begin{array}{l}\text { Category } 2 \text { low-rise exclusive } \\
\text { residential districts }\end{array}$ & 60 & 200 & 3.33 \\
\hline & $\begin{array}{l}\text { Category } 1 \text { medium-to-high-rise } \\
\text { exclusive residential districts }\end{array}$ & 60 & 200 & 3.33 \\
\hline & \multirow{2}{*}{$\begin{array}{l}\text { Category } 2 \text { medium-to-high-rise } \\
\text { exclusive residential districts }\end{array}$} & 60 & 200 & 3.33 \\
\hline & & 60 & 300 & 5.00 \\
\hline & Category 1 residential districts & 60 & 300 & 5.00 \\
\hline & \multirow{2}{*}{ Category 2 residential districts } & 60 & 300 & 5.00 \\
\hline & & 60 & 400 & 6.67 \\
\hline & \multirow{2}{*}{ Quasi-residential districts } & 60 & 300 & 5.00 \\
\hline & & 60 & 400 & 6.67 \\
\hline \multirow{11}{*}{ 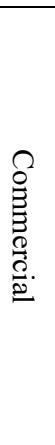 } & \multirow{3}{*}{ Neighborhood commercial districts } & 80 & 200 & 2.50 \\
\hline & & 80 & 300 & 3.75 \\
\hline & & 80 & 400 & 5.00 \\
\hline & \multirow{8}{*}{ Commercial districts } & 80 & 300 & 3.75 \\
\hline & & 80 & 400 & 5.00 \\
\hline & & 80 & 500 & 6.25 \\
\hline & & 80 & 600 & 7.50 \\
\hline & & 80 & 700 & 8.75 \\
\hline & & 80 & 800 & 10.00 \\
\hline & & 80 & 900 & 11.25 \\
\hline & & 80 & 1000 & 12.50 \\
\hline \multirow{3}{*}{ 志 } & \multirow{3}{*}{ Quasi-industrial districts } & 60 & 200 & 3.33 \\
\hline & & 60 & 300 & 5.00 \\
\hline & & 60 & 400 & 6.67 \\
\hline
\end{tabular}

Table 1. Land-use zoning and maximum number of stories in Shibuya city 
district (2)). The mean SVF value for the points within quasi-residential district (1) is $63.40 \%$ and that within quasi-residential district (2) is $55.80 \%$.

Yamanote-dori Street is a 40-m-wide road within quasiresidential district (1), consisting of an approximately 24$\mathrm{m}$-wide roadway and approximately $8-\mathrm{m}$ sidewalks on both sides of the roadway. Table 1 lists the maximum number of stories, which was calculated by dividing the floor area ratio by the building coverage ratio for each use district in Shibuya city ${ }^{8}$. It is assumed that quasiresidential district (1) exhibits a high SVF value because the width of the road facing the district is wide, regardless of the fact that the maximum number of stories is 6.67 and the building height can be increased. The width of the roads within quasi-residential district (2) is approximately $10 \mathrm{~m}$, and the maximum number of stories is 5 . The SVF value in quasi-residential district (2) is lower than that in quasi-residential district (1), and we suggest that this is owing to the narrower roads compared to Yamanote-dori Street.

Generally, quasi-residential districts are often specified along an arterial road, where five or six-story buildings can be constructed. When medium-to-high-rise buildings are located on both sides of the road, it is still possible to have a mean SVF value of $60 \%$ or higher when the road width is $40 \mathrm{~m}$, and it is also possible to have an SVF value of approximately $55 \%$ when the road width is $10 \mathrm{~m}$. Next, we focus on the SVF distribution for Category 2 low-rise exclusive residential districts and similar commercial districts. Table 2 lists the ratio of the road length of the roads whose width is less than $5.5 \mathrm{~m}^{9}$ to the whole length of the roads by land-use zoning in Shibuya city. As listed in Table 2, the roads whose width is less than $5.5 \mathrm{~m}$ account for $86.52 \%$ of all the roads, and the maximum number of stories is 3.33 according to Table 1 for Category 2 low-rise exclusive residential districts. Thus, it is shown that a large part of this district consists of narrow roads lined with low-rise buildings. As listed in Table 2, the roads whose width is less than $5.5 \mathrm{~m}$ account for $36.76 \%$ of all the roads. According to Table 1, the buildings whose maximum number of stories ranges from 3.75 to 12.50 line the roads in commercial districts. As described above, even with different combinations of the road width and building height a similar SVF result is obtained for Category 2 low-rise exclusive residential districts and commercial districts. It is considered that the characteristics of the SVF are observed based on the ratio of the visible sky instead of the amount of the threedimensional space seen as the sky. Therefore, it is suggested that comparable SVF values can be set by defining the height of the buildings according to the width of the roads.

${ }^{8}$ The actual value may be smaller than the calculated value in some cases, such as the case when the restriction of the oblique line is applied or the case of the building with a setback

${ }^{9}$ Roads whose width is less than $5.5 \mathrm{~m}$ are the narrow ones without the center line.

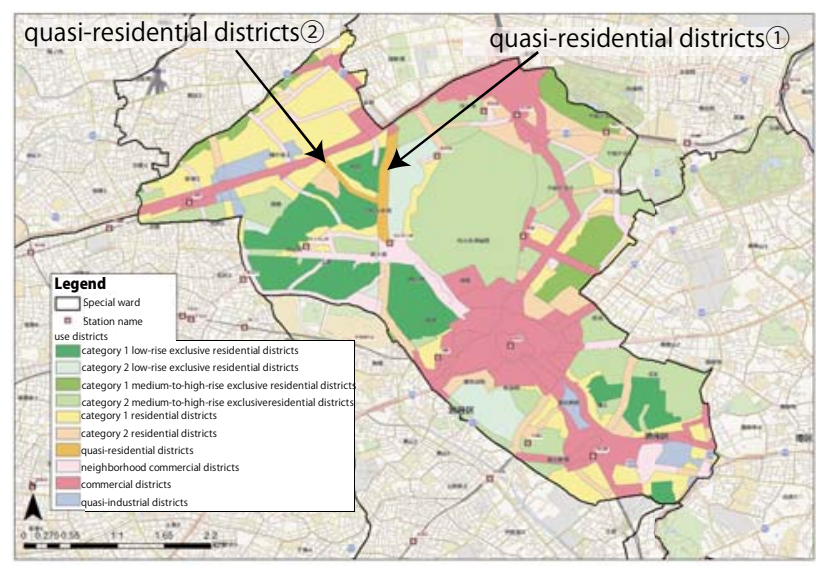

Figure 5. Land-use zoning in Shibuya city

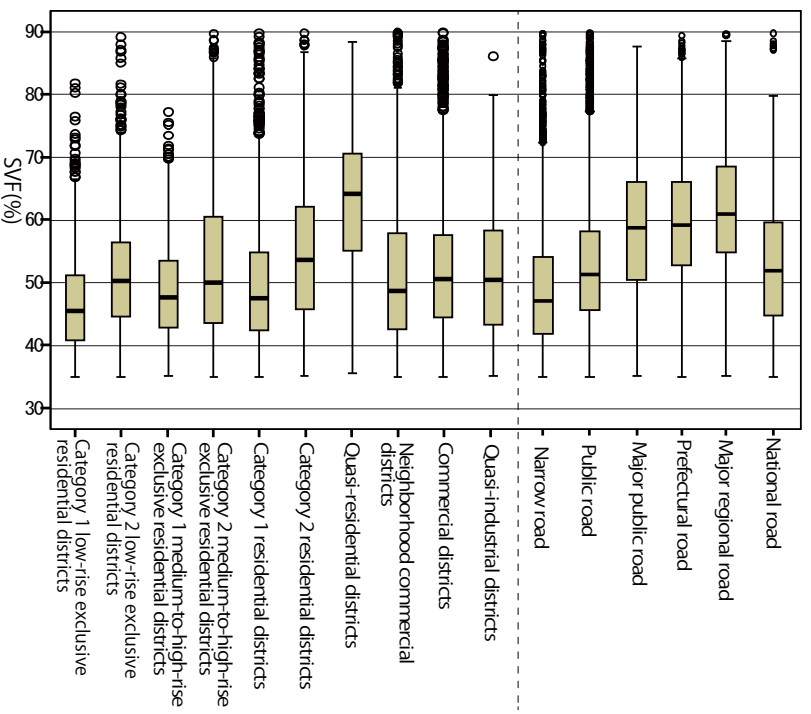

Figure 6. Box plot of the SVF by (a) land-use zoning and (b)road type

\begin{tabular}{ccc}
\hline Land-use Zoning & $\begin{array}{c}\text { Whole } \\
\text { Length } \\
(\mathrm{km})\end{array}$ & $\begin{array}{c}\text { Ratio of Roads } \\
\text { whose Width is } \\
\text { less than 5.5 m } \\
\text { to Whole Length } \\
(\%)\end{array}$ \\
\hline $\begin{array}{c}\text { Category 1 low-rise exclusive } \\
\text { residential districts }\end{array}$ & 34.28 & 85.70 \\
$\begin{array}{c}\text { Category 2 low-rise exclusive } \\
\text { residential districts }\end{array}$ & 11.25 & 86.52 \\
$\begin{array}{c}\text { Category 1 medium-to-high-rise } \\
\text { exclusive residential districts }\end{array}$ & 9.65 & 94.32 \\
$\begin{array}{c}\text { Category 2 medium-to-high-rise } \\
\text { exclusive residential districts }\end{array}$ & 35.06 & 62.89 \\
$\begin{array}{c}\text { Category 1 residential districts } \\
\text { Category 2 residential districts } \\
\text { Quasi-residential districts }\end{array}$ & 48.65 & 86.05 \\
Neighborhood commercial & 19.30 & 41.94 \\
$\quad$ districts & 8.86 & 37.20 \\
$\quad$ Commercial districts & 73.01 & 36.74 \\
$\quad$ Quasi-industrial districts & 5.65 & 76.13 \\
\hline
\end{tabular}

Table 2. Ratio of the road length by road width to the entire length of the roads by land-use zoning in Shibuya city 
Overall, the mean SVF value ranges from $45 \%$ to $55 \%$ in all the districts, except quasi-residential districts, regardless of whether these buildings are residential, commercial, or industrial. This indicates that a certain level of environment is secured in each of the districts. Tables 1 and 2 indicate that the current form restriction of land-use zoning specifies a lower building height in districts for residential use, where there are numerous narrow roads, and a higher height in districts near a wide road. Considering the above, it can be stated that the concept of form restriction in land-use zoning contributes to a comfortable urban space.

The scenarios mentioned earlier can be explained based on the ratio of the road width and building height, and it is considered that the SVF is defined at a certain degree with $\mathrm{D} / \mathrm{H}^{10}$ on a straight road. However, it is not possible to explain the road characteristics with $\mathrm{D} / \mathrm{H}$ at places such as a three-way junction and roads with the significant change in the width. Contrastingly, some of the roads in Shibuya city are arterial roads, which are straight, and there are dead ends and curved roads as well in the alleys. An analysis using the SVF can be expected to be performed for these places. The SVF calculated with the method is affected by the overhead line in a narrow road such as an alley, and there is an error in the value of the SVF; therefore, we did not perform the analysis for this case. In this regard, we work on the improvement of the method for further research.

\subsection{Road Types and SVF}

The tendency for a high SVF value in quasi-residential districts with wide roads is indicated in the previous section. It is considered that there are some cases where the SVF is influenced by the road width. Therefore, we discuss the relation between the road types and SVF.

As listed in Table 3, the roads are categorized into six types: national roads, major regional roads, prefectural roads, major public roads, public roads, and narrow roads. First, the SVF is visualized using all the data from Shibuya city for the various road types. A box plot is shown in Figure 6. First, the highest mean SVF value is observed in the major regional road. The major regional road includes Yamanote-dori Street and Meiji-dori Street, which have wide widths. Second, a mean SVF value of approximately $58 \%$ is observed in the major public road and prefectural road. These include Inokashira-dori Street and Nakano-dori Street, and these appear to be wide roads. Third, the mean SVF value is lowest in the narrow roads. Finally, despite the fact that national roads are wide, the mean SVF value is approximately $52 \%$. We consider this to be a unique scenario where the national road is located along the Metropolitan Expressway and an overhead line is seen above. Moreover, the low SVF value is different from that expected in an ordinary national road.

As explained above, the wide roads tend to exhibit high SVF values. Although high-rise buildings are located along these roads, it is possible to keep the SVF high by

\footnotetext{
${ }^{10}$ Ratio between the road width and surrounding building height
}

ensuring a road is sufficiently wide.

\begin{tabular}{|c|l|}
\hline Road Types & \multicolumn{1}{|c|}{ Definition } \\
\hline National road & $\begin{array}{l}\text { Roads whose routes are specified according to } \\
\text { article 5 of the Road Traffic Act }\end{array}$ \\
\hline $\begin{array}{c}\text { Major } \\
\text { regional road }\end{array}$ & $\begin{array}{l}\text { Major prefectural roads and city roads specified } \\
\text { by the Ministry of Land, Infrastructure, and } \\
\text { Transport according to article 56 of Road } \\
\text { Traffic, out of the prefectural roads defined by } \\
\text { article 7 of the Road Traffic Act. }\end{array}$ \\
\hline $\begin{array}{c}\text { Prefectural } \\
\text { road }\end{array}$ & $\begin{array}{l}\text { Prefectural roads defined by article 7 of the } \\
\text { Road Traffic Act, excluding major regional } \\
\text { roads }\end{array}$ \\
\hline $\begin{array}{c}\text { Major public } \\
\text { road }\end{array}$ & $\begin{array}{l}\text { Roads excluding highways, national roads, } \\
\text { major regional roads, specified city roads, and } \\
\text { prefectural roads, whose number of lanes is two } \\
\text { or more and which connect major roads } \\
\text { However, one-lane roads are included in some } \\
\text { cases }\end{array}$ \\
\hline Public road & $\begin{array}{l}\text { Roads whose effective width is between 5.5 m } \\
\text { and 13 m (5.5 m inclusive and 13 m exclusive) }\end{array}$ \\
\hline Narrow road & Roads whose effective width is less than 5.5 m \\
\hline
\end{tabular}

Table 3. Definitions of the road types

\section{Visualization of SVF Change}

We describe the method for calculating the change in the SVF. Information about the road network from GSV in Shibuya city was extracted using the Google Maps API. The number of links connecting adjacent points in GSV was 34471 . The difference in the SVF between both ends of these links was calculated as an absolute value, which was defined as the SVF change. For the SVF change acquired in Shibuya city, the mean value was $7.68 \%$, median was $3.95 \%$, and standard deviation was $9.90 \%$.

All the data on the SVF change acquired throughout Shibuya city and the histogram are shown in Figure 7. The SVF change is less than $5 \%$ in numerous places (Figure 7), and no sudden SVF changes are seen at the 10-m intervals. We observe that the tendency for the SVF change to be noticeable is more likely near an intersection. There are some places where the SVF changes owing to a difference in the elevation. It remains a future research task to show how such points are distributed throughout a city.

\section{Conclusions}

A method that calculates the SVF over a large scale and in detail (Nishio et al., 2014) is used for visualizing its distribution in Shibuya city. The relation between the SVF and characteristics of the area is analyzed. Regarding the relation between the SVF and land-use zoning, it is confirmed that the SVF value is high in quasi-residential districts located along relatively wide roads. As for the relation between the SVF and road types, a high SVF value tends to be observable along highgraded trunk roads.

Although high-rise buildings may be located along a road, it is possible to keep SVF high by ensuring the road is sufficiently wide. Thus, comparable SVF values can be 
obtained by defining the heights of the buildings according to the width of the roads. According to the Building Standards Act of Japan, presently, the floor area ratio and restriction of the oblique line are defined as the form restriction by land-use zoning. However, the results indicate that the restriction and easing defined with the SVF are also effective.

Changes in the SVF are visualized. The relation between the SVF and physiographic factors should be examined quantitatively while accounting for factors such as the road width, road shape, and difference in the height after the method is improved. This remains a future research task.

\section{References}

Asada, T. and Kameyama, S. (2016). Exhaustive Analysis of Local Landscape for Wide-Area Using Panorama Image of Google Street View. Journal of Japan Society of Civil Engineers D3, 72(5), I_383-I_392.

Liang, J., Gong, J., Sun, J., Zhou, J., Li, W., and Li, Y. (2017). Automatic Sky View Factor Estimation from Street View Photographs-A Big Data Approach. Remote Sens., 9(5), 411.

Li, X., Ratti, C., and Seiferling, I. (2017). Mapping Urban Landscapes Along Streets Using Google Street View, International Cartographic Conference, ICACI 2017: Advances in Cartography and GI Science, 341-356.

Middel, A., Lukasczyk, J., Maciejewski, R., Demuzere, M., and Roth, M. (2018). Sky View Factor Footprints for
Urban Climate Modeling, Urban Climate, 25, 120-134.

Ministry of the Environment. (2003). Survey Study Business Report on the Environmental Impacts of the 2002 Heat Island Phenomenon.

Nishio, S. and Ito, F. (2014). Proposal for Sky Factor Calculation System Using Panoramic Image of Google Street View Part 2: The Improvement of The System and Its Availability. Papers and Proceedings of the Geographic Information Systems Association of Japan, 23, E-2-1.

Nishio, S. and Ito, F. (2015). The Effect of Sky Factor and the Change on Impression of Townscape: With the Experiment for Evaluation Impression While Walking. Journal of Architecture, Planning and Environmental Engineering, 80 (710), 907-914.

Takei, M. and Ohara, M. (1977). Experimental Study on Measurement of the Sense of Oppression by a Building: (Part 2) Selection Process of the Physical Scale and Proposal of the Equation for Estimating the Sense of Oppression caused by a Building in Housing Area, Transactions of Architectural Institute of Japan, 262, 103-113.

Zeng, L., Lu, J., Li, W., and Li, Y. (2018). A Fast Approach for Large-Scale Sky View Factor Estimation Using Street View Images. Building and Environment, $135,74-84$.

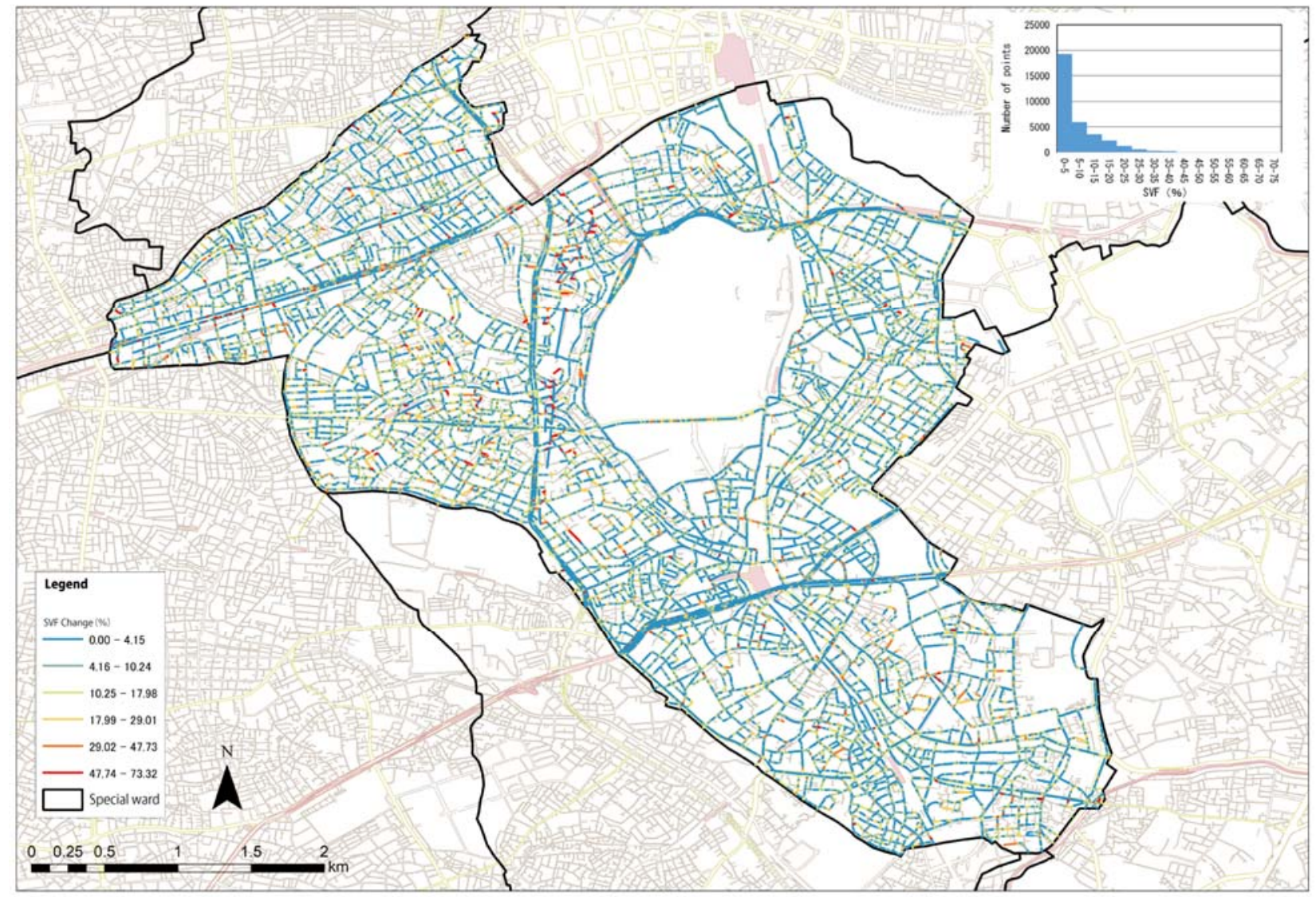

Figure 7. Visualization and histogram of the SVF changes throughout Shibuya city 